\title{
TESS first look at evolved compact pulsators
}

\section{Asteroseismology of the pulsating helium-atmosphere white dwarf TIC 257459955}

\author{
Keaton J. Bell ${ }^{1,2, \star}$, Alejandro H. Córsico ${ }^{3,4}$, Agnès Bischoff-Kim ${ }^{5}$, Leandro G. Althaus ${ }^{3,4}$, Paul A. Bradley ${ }^{6}$, \\ Leila M. Calcaferro ${ }^{3,4}$, Michael H. Montgomery ${ }^{7}$, Murat Uzundag ${ }^{8}$, Andrzej S. Baran ${ }^{9}$, Zsófia Bognár ${ }^{10,11}$, \\ Stéphane Charpinet ${ }^{12}$, Hamed Ghasemi ${ }^{13}$, and J. J. Hermes ${ }^{14}$ \\ ${ }^{1}$ Max-Planck-Institut für Sonnensystemforschung (MPS), Justus-von-Liebig-Weg 3, 37077 Göttingen, Germany \\ e-mail: keatonb@uw.edu \\ 2 Department of Physics and Astronomy, Stellar Astrophysics Centre, Aarhus University, Ny Munkegade 120, 8000 Aarhus C, \\ Denmark \\ ${ }^{3}$ Grupo de Evolución Estelar y Pulsaciones, Facultad de Ciencias Astronómicas y Geofísicas, Universidad Nacional de La Plata, \\ Paseo del Bosque s/n, 1900 La Plata, Argentina \\ 4 IALP - CONICET, Argentina \\ 5 Penn State Worthington Scranton, Dunmore, PA 18512, USA \\ 6 XCP-6, MS F-699 Los Alamos National Laboratory, Los Alamos, NM 87545, USA \\ 7 Department of Astronomy, University of Texas at Austin, Austin, TX 78712, USA \\ 8 Instituto de Física y Astronomía, Universidad de Valparaiso, Gran Bretaña 1111, Playa Ancha, Valparaíso 2360102, Chile \\ 9 Uniwersytet Pedagogiczny, Obserwatorium na Suhorze, ul. Podchorążych 2, 30-084 Kraków, Polska \\ 10 Konkoly Observatory, MTA Research Centre for Astronomy and Earth Sciences, Konkoly Thege Miklós út 15-17, 1121 Budapest, \\ Hungary \\ 11 MTA CSFK Lendület Near-Field Cosmology Research Group, Hungary \\ 12 Institut de Recherche en Astrophysique et Planétologie, CNRS, Université de Toulouse, CNES, 14 avenue Edouard Belin, \\ 31400 Toulouse, France \\ 13 Department of Physics, Institute for Advanced Studies in Basic Sciences (IASBS), Zanjan 45137-66731, Iran \\ 14 Department of Astronomy, Boston University, 725 Commonwealth Ave., Boston, MA 02215, USA
}

Received 17 July 2019 / Accepted 4 October 2019

\begin{abstract}
Context. Pulsation frequencies reveal the interior structures of white dwarf stars, shedding light on the properties of these compact objects that represent the final evolutionary stage of most stars. Two-minute cadence photometry from the Transiting Exoplanet Survey Satellite (TESS) records pulsation signatures from bright white dwarfs over the entire sky.

Aims. As part of a series of first-light papers from TESS Asteroseismic Science Consortium Working Group 8, we aim to demonstrate the sensitivity of TESS data, by measuring pulsations of helium-atmosphere white dwarfs in the DBV instability strip, and what asteroseismic analysis of these measurements can reveal about their stellar structures. We present a case study of the pulsating DBV WD 0158-160 that was observed as TIC 257459955 with the two-minute cadence for 20.3 days in TESS Sector 3.

Methods. We measured the frequencies of variability of TIC 257459955 with an iterative periodogram and prewhitening procedure. The measured frequencies were compared to calculations from two sets of white dwarf models to constrain the stellar parameters: the fully evolutionary models from LPCODE and the structural models from WDEC.

Results. We detected and measured the frequencies of nine pulsation modes and eleven combination frequencies of WD 0158-160 to $\sim 0.01 \mu \mathrm{Hz}$ precision. Most, if not all, of the observed pulsations belong to an incomplete sequence of dipole $(\ell=1)$ modes with a mean period spacing of $38.1 \pm 1.0 \mathrm{~s}$. The global best-fit seismic models from both LPCODE and WDEC have effective temperatures that are $\gtrsim 3000 \mathrm{~K}$ hotter than archival spectroscopic values of $24100-25500 \mathrm{~K}$; however, cooler secondary solutions are found that are consistent with both the spectroscopic effective temperature and distance constraints from Gaia astrometry.

Conclusions. Our results demonstrate the value of the TESS data for DBV white dwarf asteroseismology. The extent of the shortcadence photometry enables reliably accurate and extremely precise pulsation frequency measurements. Similar subsets of both the LPCODE and WDEC models show good agreement with these measurements, supporting that the asteroseismic interpretation of DBV observations from TESS is not dominated by the set of models used. However, given the sensitivity of the observed set of pulsation modes to the stellar structure, external constraints from spectroscopy and/or astrometry are needed to identify the best seismic solutions.
\end{abstract}

Key words. asteroseismology - stars: oscillations - stars: variables: general - white dwarfs

\footnotetext{
* NSF Astronomy and Astrophysics Postdoctoral Fellow.

Current address: DIRAC Institute, Department of Astronomy, University of Washington, Seattle, WA 98195-1580, USA.
} 


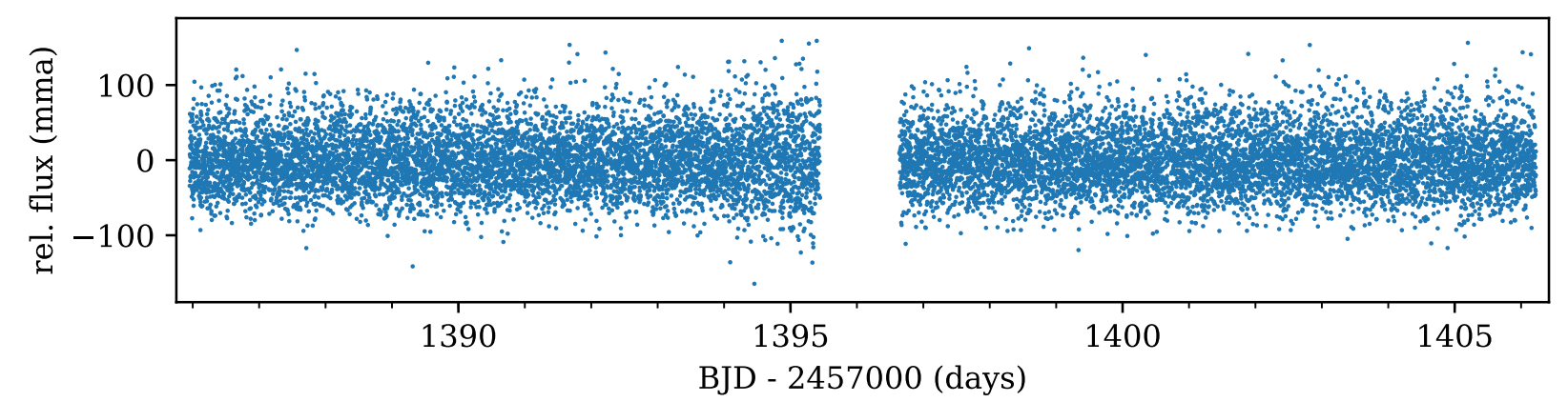

Fig. 1. Final reduced TESS Sector 3 light curve of TIC 257459955. A gap occurs at spacecraft perigee.

\section{Introduction}

The Transiting Exoplanet Survey Satellite (TESS) is a NASA mission with the primary goal of detecting exoplanets that transit the brightest and nearest stars (Ricker et al. 2014). More generally, the extensive time series photometry that TESS acquires is valuable for studying a wide variety of processes that cause stars to appear photometrically variable. One particularly powerful use for these data is to constrain the global properties and interior structures of pulsating stars with the methods of asteroseismology. Pulsating stars oscillate globally in standing waves that propagate through and are affected by the stellar interiors. Fourier analysis of the light curves of pulsating stars reveals their eigenfrequencies that can be compared to calculations from stellar models, providing the most sensitive technique for probing stellar interior structures.

The TESS Asteroseismic Science Consortium (TASC) is a collaboration of the scientific community that shares an interest in utilizing TESS data for asteroseimology research. It is organized into a number of working groups that address different classes of stars. TASC Working Group 8 (WG8) focuses on TESS observations of evolved compact stars that exhibit photometric variability, including hot subdwarfs, white dwarf stars, and pre-white dwarfs. To this goal, WG8 has proposed for all known and likely compact stars with TESS magnitudes $\lesssim 16$ to be observed at the short, two-minute cadence.

Within TASC WG8, the subgroup WG8.2 coordinates the studies of pulsating white dwarfs observed by TESS. Depending on their atmospheric compositions, white dwarfs may pulsate as they cool through three distinct instability strips: DOVs (GW Vir stars or pulsating PG 1159 stars) are the hottest and include some central stars of planetary nebulae; DBVs (V777 Her stars) have helium atmospheres that are partially ionized in the effective temperature range $32000 \gtrsim T_{\text {eff }} \gtrsim 22000 \mathrm{~K}$, driving pulsations; and DAVs (ZZ Ceti stars) pulsate when their pure-hydrogen atmospheres are partially ionized from $12500 \gtrsim T_{\text {eff }} \gtrsim 10800 \mathrm{~K}$ (at the canonical mass of $\approx 0.6 M_{\odot}$ ). Pulsations of these objects probe the physics of matter under the extreme pressures of white dwarf interiors. Since white dwarfs are the final products of $\approx 97 \%$ of Galactic stellar evolution, asteroseismic determination of their compositions and structures probes the physical processes that operate during previous evolutionary phases. See Winget \& Kepler (2008), Fontaine \& Brassard (2008), and Althaus et al. (2010) for reviews of the field of white dwarf asteroseismology, and Córsico et al. (2019) for coverage of the most recent decade of discovery in the era of extensive space-based photometry from Kepler and $\mathrm{K} 2$.

As part of the initial activities of the TASC WG8.2, we present analyses of examples of each type of pulsating white dwarf observed at two-minute cadence in the first TESS Sectors in a series of first-light papers. These follow the TASC WG8.3 first-light analysis of a pulsating hot subdwarf in TESS data from Charpinet et al. (2019). In this paper, we study the DBV pulsator WD 0158-160 (also EC 01585-1600, G 272-B2A), which was observed by TESS as target TIC 257459955 in Sector 3. Voss et al. (2007) confirm the classification of WD 0158-160 as a DB (helium-atmosphere) white dwarf from an ESO Supernova type Ia Progenitor surveY (SPY) spectrum and measure atmospheric parameters of $T_{\text {eff }}=25518 \mathrm{~K}$ and $\log g=7.875$. The more recent spectroscopic study of Rolland et al. (2018) finds a cooler best-fit model for their observations, obtaining $T_{\text {eff }}=24130 \pm 1369 \mathrm{~K}$ and $\log g=7.94 \pm 0.03$. Astrometric parallax from Gaia DR2 (Gaia Collaboration 2016, 2018) place WD 0158-160 at a distance of $68.14 \pm 0.28 \mathrm{pc}$ (BailerJones et al. 2018). This is one of the brightest DBVs known $(V=14.55 \pm 0.08 \mathrm{mag}$; Zacharias et al. 2012) and was discovered to be a variable by Kilkenny (2016). They obtained highspeed photometry on the Sutherland 1-meter telescope of the South African Astronomical Observatory over five nights, measuring ten frequencies of significant variability between 1285 $5747 \mu \mathrm{Hz}$. We aim to measure more precise pulsation frequencies from the TESS data and to compare these with stellar models to asteroseismically constrain the properties of this DB white dwarf.

\section{TESS data}

TIC 257459955 was observed at the short, two-minute cadence by TESS in Sector 3, which collected 20.3 days of useful data with a 1.12-day gap at spacecraft perigee ${ }^{1}$. Light curves from this particular Sector are shorter than the nominal 27-day duration, and the periodogram achieves a correspondingly lower frequency resolution and signal-to-noise than expected for most TESS observations. Thus, TESS's value for asteroseismology of white dwarfs observed in Sectors with longer coverage is typically greater than demonstrated in this paper.

We use the two-minute short-cadence TESS light curve of TIC 257459955 that has had common instrumental trends removed by the Pre-Search Data Conditioning Pipeline (PDC; Stumpe et al. 2012) that we downloaded from MAST ${ }^{2}$. We discard two observations that have quality flags set by the pipeline. We do not identify any additional outlying measurements that need to be removed. The final light curve contains 13450 measurements that span 20.27 days.

To remove any additional low-frequency systematics from the light curve, we divide out the fit of a fourth-order

\footnotetext{
1 See TESS Data Release Notes: http://archive.stsci.edu/ tess/tess_drn.html

2 https://archive.stsci.edu/
} 


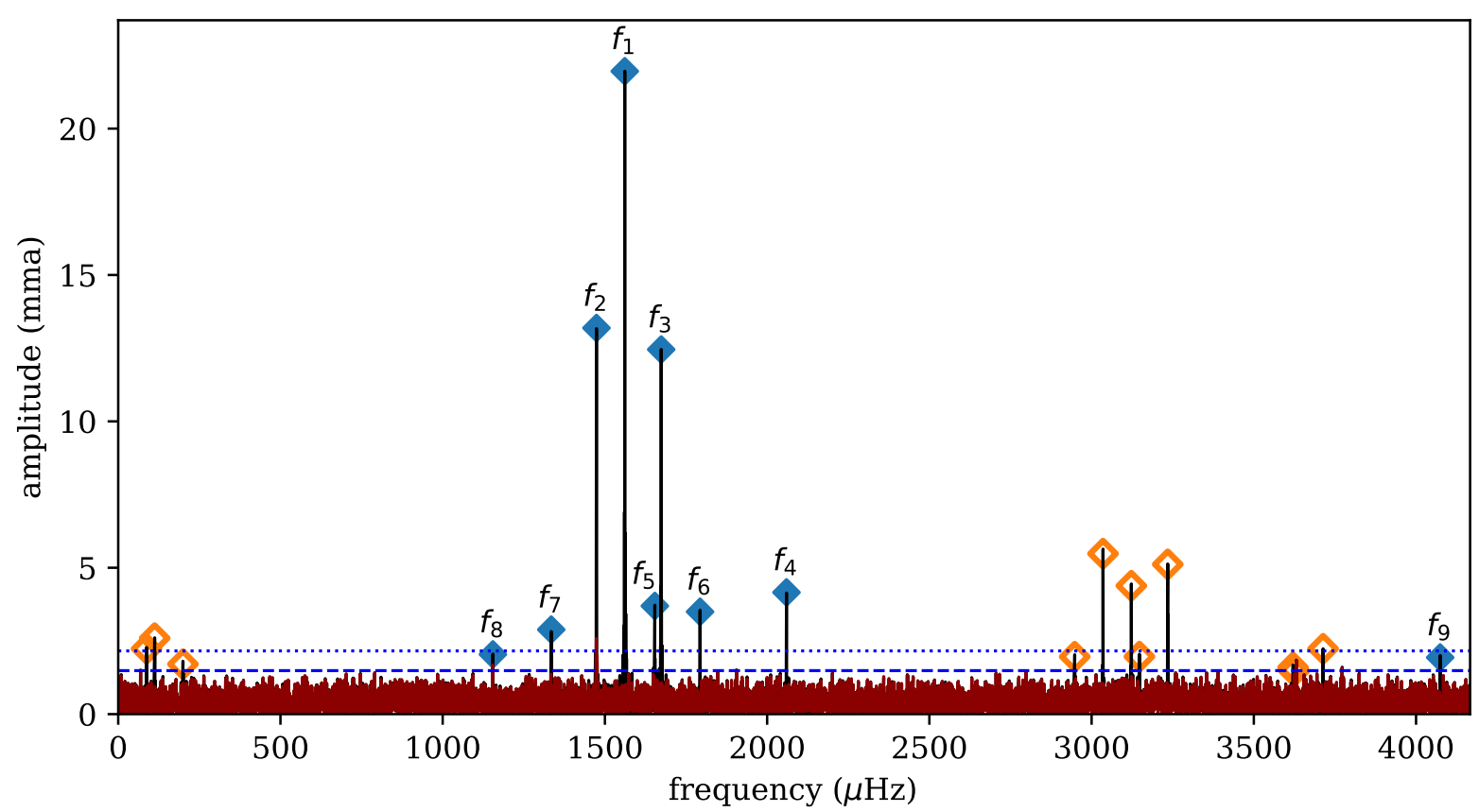

Fig. 2. Lomb-Scargle periodogram of the original TIC 257459955 light curve (black) and of the residuals after subtracting off the best fit frequency solution (red). The dotted horizontal line shows the final $0.1 \%$ false-alarm-probability (FAP) significance threshold for the residual spectrum, and the dashed line is a $0.1 \%$ FAP level for an individual frequency bin. Blue filled diamonds mark the best-fit frequencies and amplitudes of independent modes, and orange, unfilled diamonds mark combination frequencies. Independent modes are labeled with mode IDs from Table 1.

Savitzky-Golay filter with a three-day window length computed with the Python package LIGHTKURVE (Barentsen et al. 2019). This preserves the signals from pulsations that typically have periods of $\$ 20 \mathrm{~min}$ in white dwarfs. The final reduced light curve is displayed in Fig. 1, where the relative flux unit of milli-modulation amplitude (mma) equals $0.1 \%$ flux variation or one part-per-thousand. The root-mean-squared scatter of the flux measurements is $37.8 \mathrm{mma}(3.78 \%)$.

\section{Frequency solution}

Astroseismology relies on the precise determination of pulsation frequencies. We benefit from the length of the TESS data providing a high frequency resolution of $0.57 \mu \mathrm{Hz}$ (inverse of the light curve duration) without complications from aliasing that would arise from large gaps in the time series. We use the fast Lomb-Scargle implementation in astropy (Astropy Collaboration 2018) to compute periodograms of the unweighted time series photometry. We oversample the natural frequency resolution by a factor of 10 so that the periodogram peaks more accurately represent the intrinsic frequencies and amplitudes of the underlying signals. The full periodogram out to the Nyquist frequency of $4166.59 \mu \mathrm{Hz}$ is displayed in black in Fig. 2 .

Including any noise peaks in our frequency solution would adversely affect our asteroseismic inferences, so we adopt a conservative significance criterion for signal amplitudes. We test the null hypothesis that the highest peak in the periodogram is caused by pure noise by treating the observed flux measurements in the light curve as a proxy for the noise distribution. We bootstrap a significance threshold by generating 10000 pure-noise time series that sample from this distribution with replacement at the observation times of the original light curve. The 99.9th highest percentile corresponds to a false alarm probability (FAP) of $0.1 \%$ that a peak with a higher amplitude anywhere in the oversampled periodogram is caused by noise alone. We have high confidence that peaks above this threshold correspond to signifi- cant signals. For our initial periodogram, we find that peaks with amplitudes above $2.71 \mathrm{mma}$ (4.7 times the mean noise level in the periodogram ${ }^{3}$ ) have FAP $<0.1 \%$.

We adopt frequencies into our solution according to an iterative prewhitening procedure. We record the frequencies and amplitudes of every peak above our $0.1 \%$ FAP significance threshold. These provide initial values for a multi-sinusoid fit to the time series data ${ }^{4}$, which we compute with the nonlinear least-squares minimization Python package lmfit (Newville et al. 2018). Frequencies that agree within the natural frequency resolution with a sum, integer multiple, or difference between higheramplitude signals are identified as combination frequencies. These arise from a nonlinear response of the flux to the stellar pulsations (Brickhill 1992), and we enforce a strict arithmetic relationship between these combination frequencies and the parent pulsation frequencies when performing the fit. Including the combination frequencies slightly improves the measurement precision of the parent mode frequencies. Once all significant signals are included in our model and fit to the time series, we subtract off the model and repeat the process on the residuals, starting by recalculating the periodogram and bootstrapping its $0.1 \%$ FAP significance threshold. This is repeated until no further signals meet our acceptance criterion.

At this point, the periodogram still exhibits a few compelling peaks at locations where we specifically expect that signals might appear. For assessing significance in these cases, we bootstrap a different $0.1 \%$ FAP threshold for the amplitude of a peak within a single frequency bin (as opposed to considering the highest peak anywhere in the entire spectrum). We adopt signals that correspond to combinations of accepted modes

3 This matches the significance threshold advocated for by Baran et al. (2015), though they use a different method to arrive at this level, interpreting it as the threshold that yields the correct frequency determinations in $95 \%$ of random realizations.

4 Our interactive Python-based periodogram and sine-fitting code is available at https://github.com/keatonb/Pyriod 
Table 1. Pulsational frequency solution from the TESS light curve of TIC 257459955.

\begin{tabular}{lccc}
\hline \hline $\begin{array}{l}\text { Mode } \\
\text { ID }\end{array}$ & $\begin{array}{c}\text { Frequency } \\
(\mu \mathrm{Hz})\end{array}$ & $\begin{array}{c}\text { Period } \\
(\mathrm{s})\end{array}$ & $\begin{array}{c}\text { Amplitude } \\
(\mathrm{mma})\end{array}$ \\
\hline$f_{1}$ & $1561.200 \pm 0.005$ & $640.533 \pm 0.002$ & $22.0 \pm 0.4$ \\
$f_{2}$ & $1473.985 \pm 0.008$ & $678.433 \pm 0.004$ & $13.2 \pm 0.4$ \\
$f_{3}$ & $1673.490 \pm 0.008$ & $597.554 \pm 0.003$ & $12.5 \pm 0.4$ \\
$f_{4}$ & $2059.62 \pm 0.02$ & $485.527 \pm 0.006$ & $4.2 \pm 0.4$ \\
$f_{5}$ & $1653.87 \pm 0.03$ & $604.642 \pm 0.010$ & $3.7 \pm 0.4$ \\
$f_{6}$ & $1793.24 \pm 0.03$ & $557.649 \pm 0.010$ & $3.5 \pm 0.4$ \\
$f_{7}$ & $1334.64 \pm 0.04$ & $749.27 \pm 0.02$ & $2.9 \pm 0.4$ \\
$f_{8}$ & $1154.77 \pm 0.06$ & $865.97 \pm 0.04$ & $2.0 \pm 0.4$ \\
$f_{9}$ & $4074.32 \pm 0.06$ & $245.440 \pm 0.004$ & $1.9 \pm 0.4$ \\
\hline$f_{1}-f_{2}$ & $87.215 \pm 0.009$ & $11466.0 \pm 1.2$ & $2.23 \pm 0.4$ \\
$f_{3}-f_{1}$ & $112.290 \pm 0.010$ & $8905.5 \pm 0.8$ & $2.60 \pm 0.4$ \\
$f_{3}-f_{2}$ & $199.504 \pm 0.011$ & $5012.4 \pm 0.3$ & $1.71 \pm 0.4$ \\
$2 f_{1}$ & $3122.400 \pm 0.009$ & $320.267 \pm 0.001$ & $4.39 \pm 0.4$ \\
$2 f_{2}$ & $2947.971 \pm 0.015$ & $339.2164 \pm 0.0017$ & $1.97 \pm 0.4$ \\
$f_{1}+f_{2}$ & $3035.185 \pm 0.009$ & $329.4692 \pm 0.0009$ & $5.5 \pm 0.4$ \\
$f_{1}+f_{3}$ & $3234.690 \pm 0.009$ & $309.1487 \pm 0.0009$ & $5.1 \pm 0.4$ \\
$f_{1}+f_{4}$ & $3620.82 \pm 0.02$ & $276.1809 \pm 0.0018$ & $1.6 \pm 0.4$ \\
$f_{2}+f_{3}$ & $3147.475 \pm 0.011$ & $317.7150 \pm 0.0011$ & $2.0 \pm 0.4$ \\
$f_{4}+f_{5}$ & $3713.49 \pm 0.03$ & $269.289 \pm 0.002$ & $2.2 \pm 0.4$ \\
$f_{1}+f_{2}+f_{3}$ & $4708.675 \pm 0.012$ & $212.3740 \pm 0.0005$ & $1.5 \pm 0.4$ \\
\hline
\end{tabular}

Notes. The independent pulsation modes are listed first in order of decreasing amplitude, followed by all identified combination frequencies.

into our solution that exceed this lower threshold. We accept another independent pulsation mode at $1154.77 \pm 0.06 \mu \mathrm{Hz}\left(f_{8}\right.$ in Table 1) that closely matches the asymptotic mean period spacing of $\ell=1$ modes that we identify in our preliminary asteroseismic mode identification in Sect. 4.1. We also include the peak at $4074.32 \pm 0.06 \mu \mathrm{Hz}\left(f_{9}\right)$ as an intrinsic pulsation mode since it agrees with the measurement of a frequency at $4074.0 \pm 0.1 \mu \mathrm{Hz}$ from the ground-based discovery data of Kilkenny (2016) to within the periodogram frequency resolution. After prewhitening these signals, the final single-bin significance threshold is at $1.50 \mathrm{mma}^{5}$, compared to the $0.1 \%$ FAP level across the entire spectrum at $2.24 \mathrm{mma}$.

The final best-fit values for the frequencies (periods) and amplitudes of the individual sinusoids in our model are given in Table 1. The quoted errors are estimated by lmfit from the covariance matrix, and they agree with expectations from analytical formulae for the independent modes (Montgomery \& O'Donoghue 1999). In Fig. 2, the dotted line indicates the final full-spectrum $0.1 \%$ FAP significance threshold and the dashed line marks the lower per-bin threshold. These values are indicated by diamond markers (independent modes in filled blue and combination frequencies in unfilled orange). The periodogram of the final residuals is displayed in red. The measured amplitudes will generally be lower than the intrinsic disk-integrated amplitudes due to smoothing from the two-minute exposures. The intrinsic frequency of the combination $f_{1}+f_{2}+f_{3}$ is above the observational Nyquist frequency, so we mark the corresponding alias peak near $3624.5 \mu \mathrm{Hz}$.

There remain conspicuous low-amplitude peaks in the prewhitened periodogram that are adjacent to the $f_{2}$ and $f_{8}$

\footnotetext{
5 The peak corresponding to the sub-Nyquist alias of the $f_{1}+f_{2}+f_{3}$ combination frequency exceeded this threshold even though the best-fit amplitude in the final solution is lower.
}

frequencies. These are likely caused by these signals exhibiting slight amplitude or phase variations during the TESS observations. The best-fit frequency values in Table 1 correspond to the highest and central peaks of each mode's power that best represent the intrinsic pulsation frequencies, though the measured amplitudes may be less than the instantaneous maximum amplitudes of these signals during the observations.

\section{Asteroseismic analyses}

As a collaborative effort of the TASC WG8.2, all members with asteroseismic tools and models suited for this data set were invited to contribute their analyses. Two groups submitted full asteroseismic analyses to this effort, which we present in this section. This is the first direct comparison between asteroseismic analyses of the La Plata and Texas groups. By including multiple analyses, we aim to assess the consistency of asteroseismic inferences for pulsating DBVs that utilize different models and methods.

Owing to the quality of the space-based data, the measurements of pulsation mode frequencies presented in Table 1 are reliably accurate and extremely precise. Both analyses that follow aim to interpret this same set of pulsation frequency measurements. Certainly the sensitivity of the set of modes detected to the detailed interior structure is a primary limitation on our ability to constrain the properties of this particular DBV.

The combination frequencies are not considered in these analyses, since these are not eigenfrequencies of the star and do not correspond to the pulsation frequencies calculated for stellar models. This highlights the importance of identifying combination frequencies as such; erroneously requiring a model frequency to match a combination frequency would derail any asteroseismic inference.

\subsection{Preliminary mode identification}

Identifying common patterns in the pulsation spectrum can guide our comparison of the measured frequencies to stellar models. Gravity $(g)$-mode pulsations of white dwarfs are non-radial oscillations of spherical harmonic eigenfunctions of the stars. We observe the integrated light from one hemisphere of a star, so geometric cancellation effects (Dziembowski 1977) typically restrict us to detecting only modes of low spherical degree, $\ell=1$ or 2 (modes with one or two nodal lines along the surface). Modes can be excited in a sequence of consecutive radial orders, $k$, for each $\ell$. In the asymptotic limit $(k \gg \ell)$, gravity modes of consecutive radial overtone are evenly spaced in period (Tassoul et al. 1990), following approximately

$\Pi_{\ell, k} \approx \Delta \Pi_{\ell}^{a} k+\epsilon=\frac{\Pi_{0}}{\sqrt{\ell(\ell+1)}} k+\epsilon$,

where $\Delta \Pi_{\ell}^{a}$ is the period spacing, $\Pi_{0}$ and $\epsilon$ are constants.

We searched for a constant period spacings in the data of TIC 257459955 using the Kolmogorov-Smirnov (K-S; see Kawaler 1988), the inverse variance (I-V; see O'Donoghue 1994) and the Fourier Transform (F-T Handler et al. 1997) significance tests. In the $\mathrm{K}-\mathrm{S}$ test, the quantity $Q$ is defined as the probability that the observed periods are randomly distributed. Thus, any uniform or at least systematically non-random period spacing in the period spectrum of the star will appear as a minimum in $Q$. In the $\mathrm{I}-\mathrm{V}$ test, a maximum of the inverse variance will indicate a constant period spacing. Finally, in the F-T test, we calculate the Fourier transform of a Dirac comb function (created from a set of observed periods), and then we plot the square 

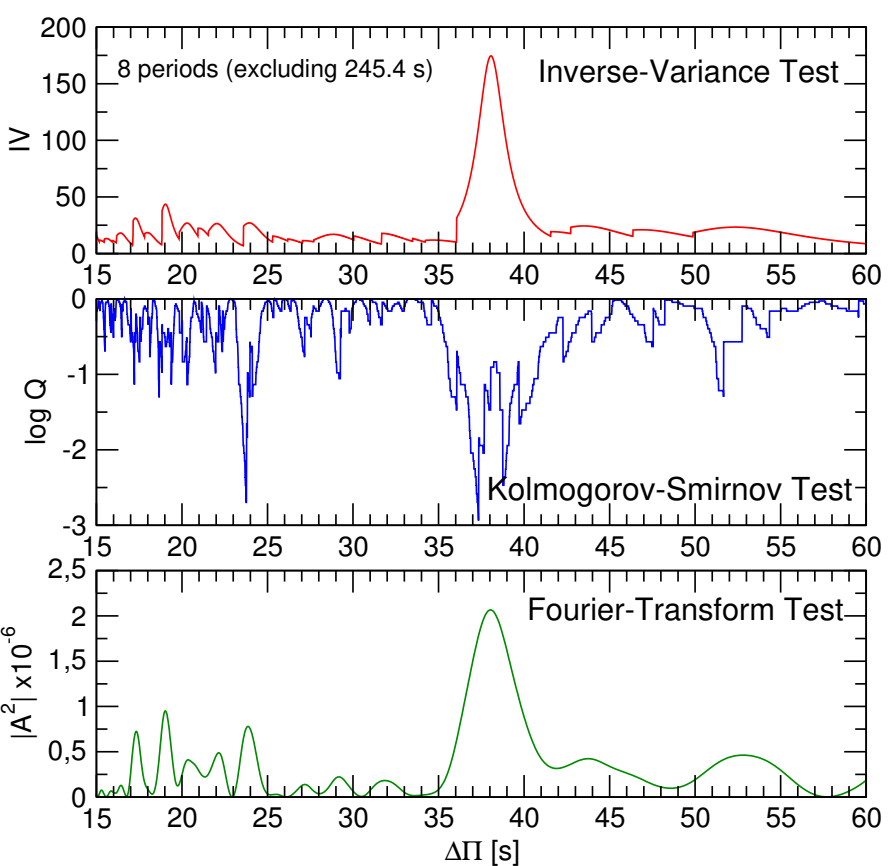

Fig. 3. $\mathrm{I}-\mathrm{V}$ (upper panel), $\mathrm{K}-\mathrm{S}$ (middle panel), and $\mathrm{F}-\mathrm{T}$ (bottom panel) significance tests to search for a constant period spacing of TIC 257459955. The tests are applied to the pulsation periods in Table 1, excluding the $245.4 \mathrm{~s}$ period that is not clearly within the asymptotic regime. See text for details.

of the amplitude of the resulting function in terms of the inverse of the frequency. And once again, a maximum in the square of the amplitude will indicate a constant period spacing. In Fig. 3 we show the results of applying the tests to the set of periods of Table 1 , excluding the short-period $f_{9}$ that is not clearly within the asymptotic $(k \gg \ell)$ regime. The three tests support the existence of a mean period spacing of about $38 \mathrm{~s}$ which corresponds to our expectations for a dipole $(\ell=1)$ sequence. For $\ell=2$, according to Eq. (1), one should find a spacing of periods of $\sim 22 \mathrm{~s}$, which is not observed in our analysis ${ }^{6}$. By averaging the period spacing derived from the three statistical tests, we found $\Delta \Pi=38.1 \pm 1.8 \mathrm{~s}$ as an initial period spacing detection.

We initially obtained a nearly identical result using a frequency solution that did not include $f_{8}$, as this peak did not exceed our independent significance threshold. Once the preliminary mode identifications were established, it became clear that $f_{8}$ is located precisely where we expect a $\ell=1$ mode given the asymptotic period spacing. This prompted us to adopt this mode into our solution for exceeding the lower, frequency-dependent significance threshold, as described in Sect. 3.

This mean period spacing of the $\ell=1$ modes cannot account for the signals at $f_{3}$ and $f_{5}$ that are separated by only $19.62 \pm 0.03 \mu \mathrm{Hz}(7.088 \pm 0.010 \mathrm{~s})$. One of these could belong to the quadrupole $(\ell=2)$ sequence. Alternatively, $f_{3}$ and $f_{5}$ could both be components of a $\ell=1$ rotational multiplet. Stellar rotation causes $2 \ell+1$ modes with different azimuthal orders, $m$, to exist for each pair of $\ell$ and $k$ (where $m$ is as integer between $-\ell$ and $\ell$ ). These are separated evenly in frequency by an amount proportional to the stellar rotation rate (e.g., Cox 1984), though many may not be excited to observable amplitude. The frequency separation between $f_{3}$ and $f_{5}$ is within the range of rotational splittings of $\ell=1$ modes detected from other pulsating white

\footnotetext{
6 There is an indication of a $\Delta \Pi \sim 24 \mathrm{~s}$, that is a bit longer than the
} prediction for $\ell=2(\Delta \Pi \sim 22 \mathrm{~s})$, but only from the $\mathrm{K}-\mathrm{S}$ test.
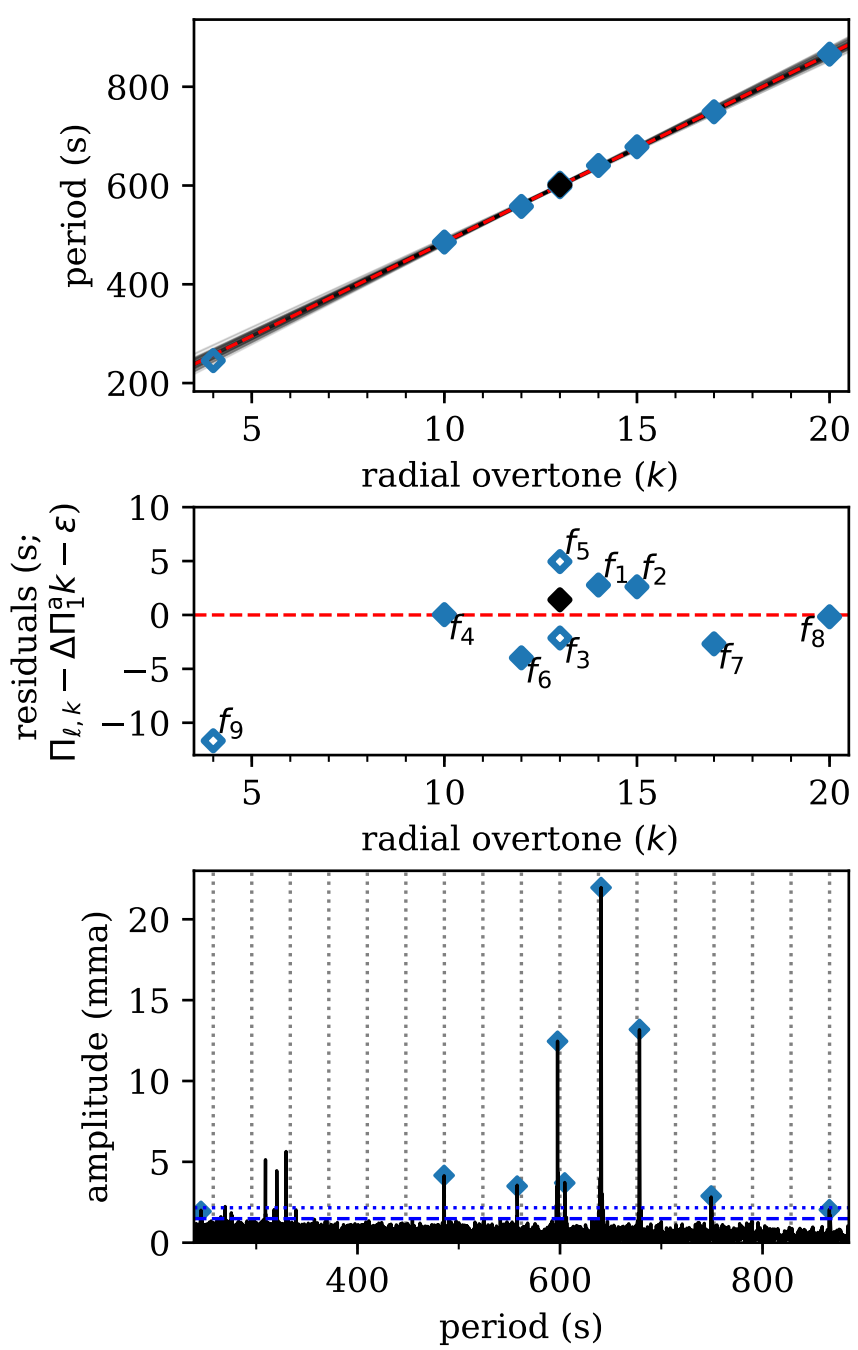

Fig. 4. Top: independent pulsation periods of the star plotted versus radial overtone number $k$. The least-squares line fit to $k>5$ (using the mean period of $f_{3}$ and $f_{5}$ for $k=13$; black) indicates a roughly constant period spacing consistent with dipole $\ell=1$ modes. The transparent gray lines represent fits to the perturbed periods assuming different observed azimuthal orders, $m$ (see text). Middle: residuals of the linear fit shows evidence of a possible mode trapping cycle. We label the mode IDs from Table 1. Bottom: pulsation spectrum in period space with the dotted vertical lines indicating the expected locations of $\ell=1$ modes from the asymptotic pulsation theory given our least-squares fit parameters.

dwarfs in space-based data (e.g., Hermes et al. 2017a). We leave the exploration of alternate interpretations of these modes up to the individual analyses that follow.

The top panel of Fig. 4 displays a least-squares fit of a line (Eq. (1)) through the periods measured for the independent modes listed in Table 1, given the preliminary period spacing detected from our initial statistical tests. The modes follow a pattern that is consistent with an incomplete $\ell=1$ sequence, though four consecutive modes are detected. The absolute radial overtone numbers, $k$, were obtained from the best period-by-period fits from both sets of models described in Sects. 4.2 and 4.3. We exclude the mode $f_{9}$ from the fit because its low radial order $(k=4)$ is furthest from the asymptotic regime. Repeating the fit using alternatively $f_{3}$ and $f_{5}$ for the $k=13, m=0$ mode has a negligible effect on the best-fit parameters. The measured periods are weighted equally in the fits since uncertainty in the azimuthal order, $m$, and physical departures from even period 
spacing likely dominate over the tiny measurement errors ${ }^{7}$ in the residuals. Using the mean period of $f_{3}$ and $f_{5}$ for $k=13$, the bestfit line has $\Delta \Pi_{1}^{a}=38.1 \pm 0.3 \mathrm{~s}$ and $\epsilon=105 \pm 5 \mathrm{~s}$ (Eq. (1)). This is consistent with the value determined from the three significance tests applied directly to the period list, but the uncertainties are underestimated because they do not account for the $m$ ambiguity. We assess our actual uncertainty by repeating fits to 1000 permutations of the periods, each time assigning every observed mode a random $m \in\{-1,0,1\}$ and then correcting to the intrinsic $m=0$ value with an assumed rotational splitting of either $f_{3}-f_{5}$ or half that value. Some representative fits are shaded in the background of Fig. 4. The standard deviation of best-fit slopes is $0.9 \mathrm{~s}$, which we add in quadrature to the fit uncertainty for a final measured $\ell=1$ asymptotic period spacing of $\Delta \Pi_{1}^{a}=38.1 \pm 1.0 \mathrm{~s}$.

The middle panel of Fig. 4 displays the residuals of the measured periods about this fit. We recognize an apparent oscillatory pattern in the residuals with a cycle length of $\Delta k \approx 6$, which could correspond to the mode trapping effect of "sharp" localized features in the stellar structure (as detected in other DBVs, e.g., Winget et al. 1994). These deviations from a strictly even period spacing may provide asteroseismic sensitivity to the location of the helium layer boundary or to chemical composition transitions in the core. The pulsation spectrum is displayed in units of period in the bottom panel of Fig. 4, with the expected locations of the $\ell=1$ modes for even period spacing indicated.

\subsection{Analysis from the La Plata group}

In our first analysis, we begin by assessing the stellar mass of TIC 257459955 following the methods described in several papers by the La Plata group on asteroseismic analyses of GW Vir stars and DBV stars (see, for instance, Córsico et al. 2007).

We first derive the "spectroscopic" stellar mass of TIC 257459955 from the $T_{\text {eff }}$ and $\log g$ values and appropriate evolutionary tracks. We adopt the values $T_{\text {eff }}=25518 \pm 1000 \mathrm{~K}$ and $\log g=7.875 \pm 0.06$ from Voss et al. $(2007)^{8}$, and employ the DB white dwarf evolutionary tracks from Althaus et al. (2009) produced with the LPCODE evolutionary code. These evolutionary tracks have been employed in the asteroseismic analyses of the DBV stars KIC 8626021 (Córsico et al. 2012), KUV 05134+2605 (Bognár et al. 2014), and PG 1351+489 (Córsico et al. 2014). The sequences of DB white dwarf models have been obtained taking into account a complete treatment of the evolutionary history of progenitors stars, starting from the zero-age main sequence (ZAMS), through the thermally pulsing asymptotic giant branch (TP-AGB) and born-again (VLTP; very late thermal pulse) phases to the domain of the PG 1159 stars, and finally the DB white dwarf stage. As such, they are characterized by evolving chemical profiles consistent with the prior evolution. We varied the stellar mass and the effective temperature in our model calculations, while the He content, the chemical structure at the $\mathrm{CO}$ core, and the thickness of the chemical interfaces were fixed by the evolutionary history of progenitor objects. These employ the ML2 prescription of convection with the mixing length parameter, $\alpha$, fixed to 1 (Bohm \& Cassinelli 1971; Tassoul et al. 1990). In Fig. 5 we show the evolutionary tracks along with the location of all the DBVs known to date (Córsico et al. 2019). We derive a new value of the spectroscopic mass for this star on the basis of this set of evolutionary models.

\footnotetext{
The error bars on the period measurements are much smaller than the points in Fig. 4.

8 We adopt uncertainties $\sigma_{T_{\text {eff }}}=1000 \mathrm{~K}$ and $\sigma_{\log g}=0.06$ as nominal errors of $T_{\text {eff }}$ and and $\log g$.
}

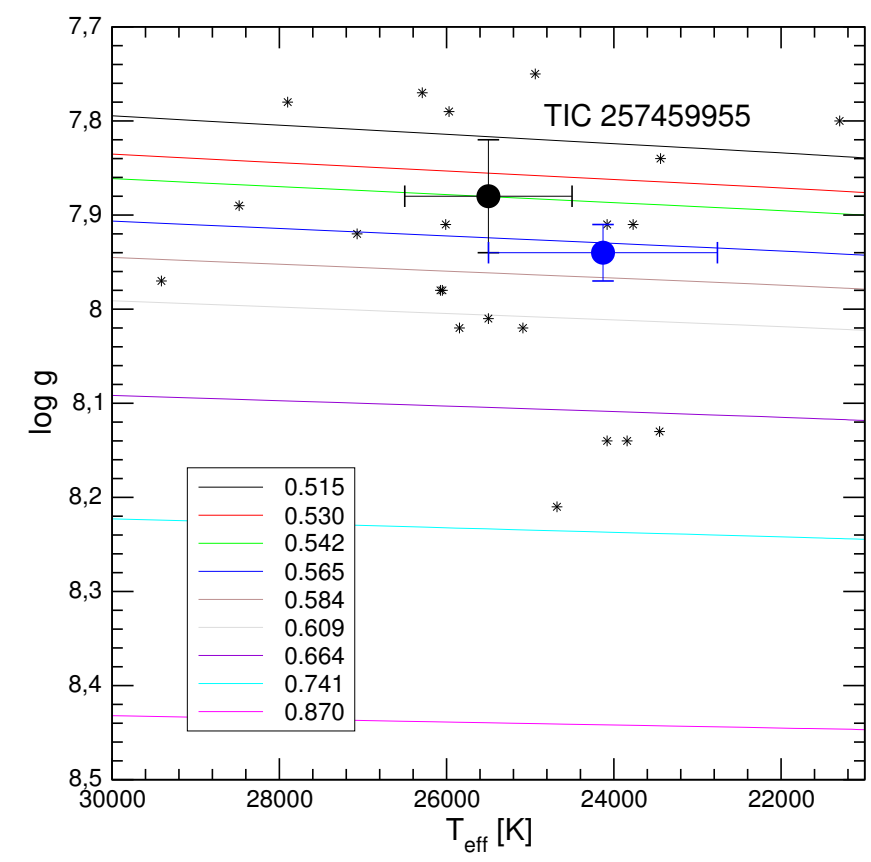

Fig. 5. Location of the known DBV stars on the $T_{\text {eff }}-\log g$ diagram (black star symbols) according to the compilation by Córsico et al. (2019). The DB white dwarf evolutionary tracks are depicted with different colors according to the stellar mass. Stellar masses are in solar units. The location of TIC 257459955 as given by spectroscopy according to Voss et al. (2007) and Rolland et al. (2018) are highlighted with black and blue circles and error bars. The stellar mass derived from linear interpolation results $M_{\star}=0.542_{-0.025}^{+0.028} M_{\odot}$ using the data from Voss et al. (2007), and $M_{\star}=0.570_{-0.011}^{+0.009} M_{\odot}$ employing the data from Rolland et al. (2018).

This is relevant because this same set of DB white dwarf models is used below to derive the stellar mass from the period spacing of TIC 257459955. By linear interpolation we obtain an estimate of the spectroscopic mass of $M_{\star}=0.542_{-0.025}^{+0.028} M_{\odot}$ when we use the spectroscopic parameters from Voss et al. (2007), and $M_{\star}=0.570_{-0.011}^{+0.009} M_{\odot}$ if we adopt the spectroscopic parameters from Rolland et al. (2018).

In Sect. 4.1, we identified an incomplete dipole $(\ell=1)$ sequence of gravity modes with high radial order $k$ (long periods) with consecutive modes $(|\Delta k|=1)$ that are nearly evenly separated in period by $\Delta \Pi_{1}^{a}=38.1 \pm 1.0 \mathrm{~s}$. This follows our expectations from the asymptotic theory of non-radial stellar pulsations given by Eq. (1), where

$\Pi_{0}=2 \pi^{2}\left[\int_{r_{1}}^{r_{2}} \frac{N}{r} \mathrm{~d} r\right]^{-1}$,

$N$ being the Brunt-Väisälä frequency, one of the critical frequencies of non-radial stellar pulsations. In principle, the asymptotic period spacing or the average of the period spacings computed from a grid of models with different masses and effective temperatures can be compared with the mean period spacing exhibited by the star to infer the value of the stellar mass. These methods take full advantage of the fact that the period spacing of DBV stars primarily depends on the stellar mass and the effective temperature, and very weakly on the thickness of the He envelope (see, e.g., Tassoul et al. 1990).

We assessed the average period spacings computed for our models as $\overline{\Delta \prod_{\ell=1}}=(n-1)^{-1} \sum_{k} \Delta \Pi_{k}$, where the "forward" period spacing is defined as $\Delta \Pi_{k}=\Pi_{k+1}-\Pi_{k}$ ( $k$ being the 


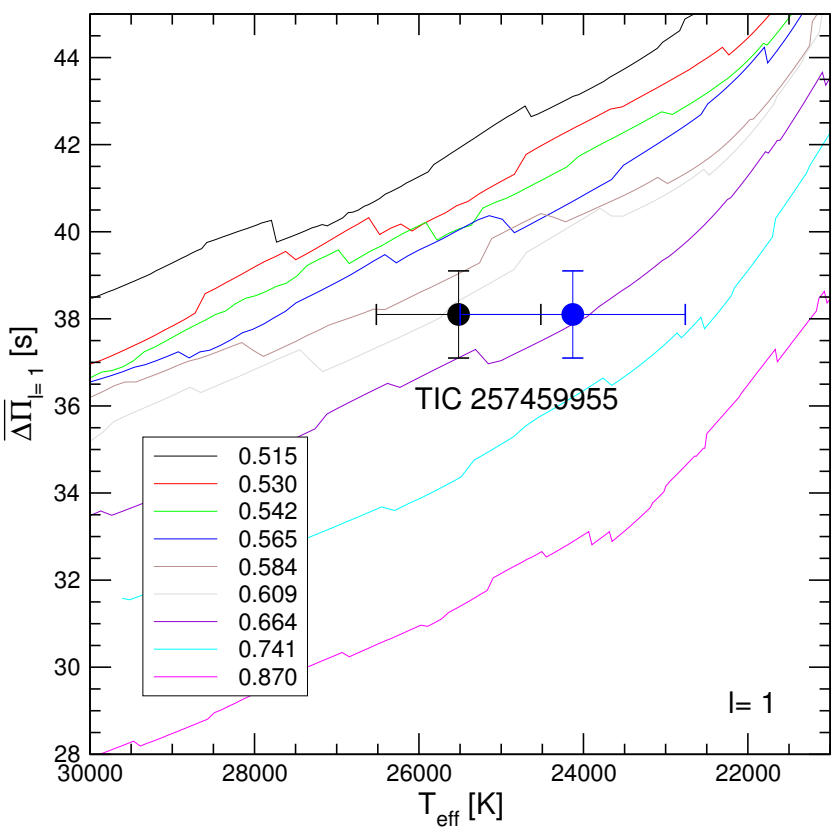

Fig. 6. Dipole $(\ell=1)$ average of the computed period spacings, $\overline{\Delta \Pi_{\ell=1}}$, assessed in a range of periods that includes the periods observed in TIC 257459955, shown as solid curves of different colors for different stellar masses. The location of TIC 257459955 when we use the effective temperatures derived by Voss et al. (2007) and Rolland et al. (2018) are highlighted with black and blue circles. We include the error bars associated to the uncertainties in $\Delta \Pi$ and $T_{\text {eff }}$. The stellar mass derived from linear interpolation is $M_{\star}=0.621_{-0.052}^{+0.057} M_{\odot}\left(M_{\star}=0.658_{-0.074}^{+0.106} M_{\odot}\right)$ by using the $T_{\text {eff }}$ derived by Voss et al. (2007) and Rolland et al. (2018).

radial order) for $\ell=1$ modes and $n$ is the number of theoretical periods considered from the model. The theoretical periods were computed with the LP-PUL pulsation code (Córsico \& Althaus 2006). For TIC 257459955, the observed mode periods are $\Pi_{k} \in[245,866] \mathrm{s}$. In computing the average period spacings for the models, however, we have considered the range $[470,1400] \mathrm{s}$, that is, we excluded short periods that are probably outside the asymptotic regime. We also adopt a longer upper limit of this range of periods in order to better sample the period spacing of modes within the asymptotic regime. In Fig. 6 we show the run of the average of the computed period spacings $(\ell=1)$ in terms of the effective temperature for our DBV evolutionary sequences, along with the observed period spacing for TIC 257459955. As can be appreciated from the figure, the greater the stellar mass, the smaller the computed values of the average period spacing. By means of a linear interpolation of the theoretical values of $\overline{\Delta \Pi_{\ell=1}}$, the measured $\Delta \Pi$ and spectroscopic effective temperatures yield stellar masses of $M_{\star}=0.621_{-0.052}^{+0.057} M_{\odot}$ by using the $T_{\text {eff }}$ value from Voss et al. (2007) and $M_{\star}=0.658_{-0.074}^{+0.106} M_{\odot}$ by employing the $T_{\text {eff }}$ estimate from Rolland et al. (2018). These stellar-mass values are higher than the spectroscopic estimates of the stellar mass.

On the other hand, if we instead fix the mass to the value derived from the spectroscopic $\log g\left(0.542-0.570 M_{\odot}\right)$, then we need to shift the model to higher effective temperature $(\simeq 28500 \mathrm{~K})$. This is the result we recover and refine in the period-to-period fitting. In this procedure we search for a pulsation model that best matches the individual pulsation periods of the star under study. The goodness of the match between the theoretical pulsation periods $\left(\Pi_{k}^{\mathrm{T}}\right)$ and the observed individual periods $\left(\Pi_{i}^{\mathrm{O}}\right)$ is assessed by using a merit function defined as:

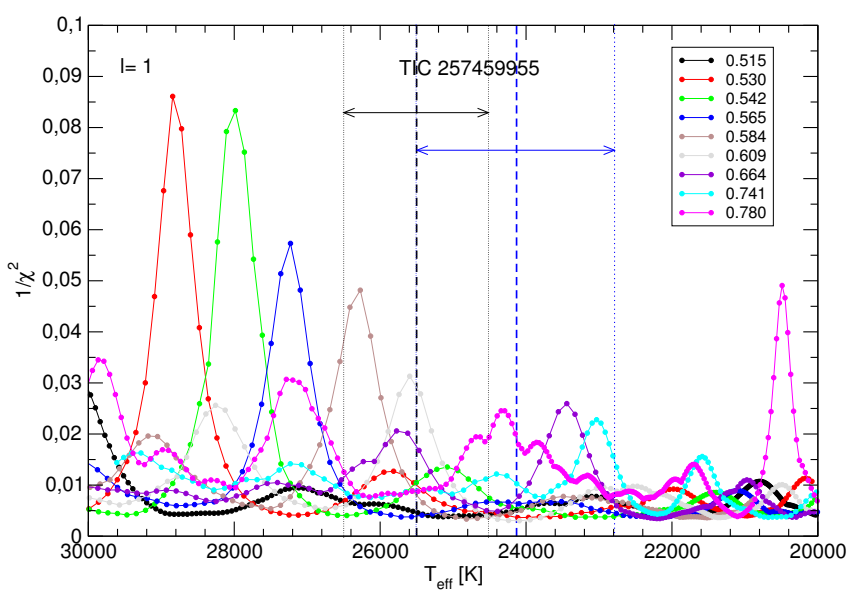

Fig. 7. Inverse of the quality function of the period fit in terms of the effective temperature for the case in which we assume that the modes are all $\ell=1$. The vertical black dashed line indicates the spectroscopic $T_{\text {eff }}$ of TIC 257459955 and the vertical dotted lines its uncertainties according to Voss et al. (2007; $\left.T_{\text {eff }}=25518 \pm 1000 \mathrm{~K}\right)$. Similarly, the blue vertical lines correspond to the spectroscopic $T_{\mathrm{eff}}$ and its uncertainties as derived by Rolland et al. (2018; $\left.T_{\text {eff }}=24130 \pm 1369 \mathrm{~K}\right)$.

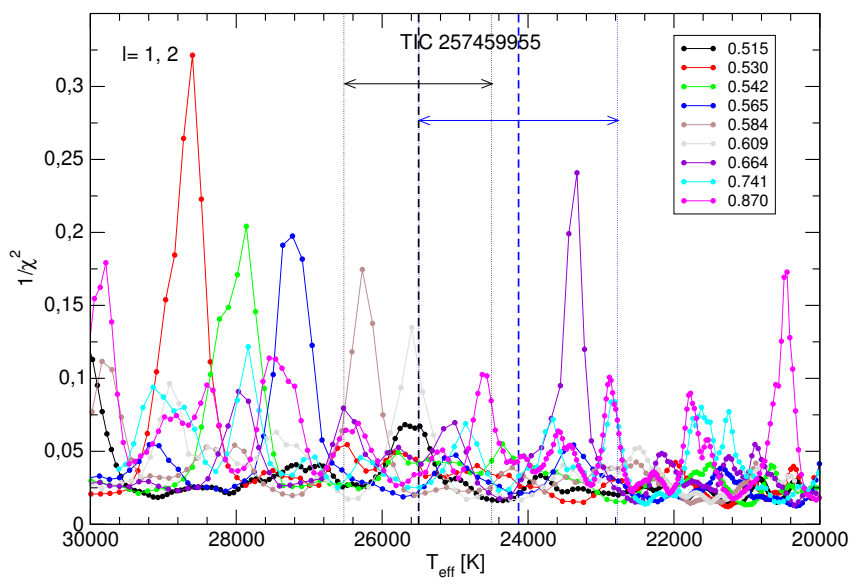

Fig. 8. Same as in Fig. 7, but for the case in which we assume a mix of $\ell=1$ and $\ell=2$ modes.

$\chi^{2}\left(M_{\star}, T_{\text {eff }}\right)=\frac{1}{m} \sum_{i=1}^{m} \min \left[\left(\Pi_{i}^{\mathrm{O}}-\Pi_{k}^{\mathrm{T}}\right)^{2}\right]$,

where $m$ is the number of observed periods. The DB white dwarf model that shows the lowest value of $\chi^{2}$, if one exists, is adopted as the global "best-fit model". We assess the function $\chi^{2}=\chi^{2}\left(M_{\star}, T_{\text {eff }}\right)$ for stellar masses in the range $\left[0.515 M_{\odot}-0.741 M_{\odot}\right]$. For the effective temperature we employ a much finer grid $\left(\Delta T_{\text {eff }} \sim 20 \mathrm{~K}\right)$ which is given by the time step adopted in the evolutionary calculations of LPCODE. We assumed that the nine pulsation periods of TIC 257459955 (Table 1) correspond to (i) modes with $\ell=1$ only, and (ii) a mix of $\ell=1$ and $\ell=2$ modes. The results are shown in Figs. 7 and 8 , in which we depict the inverse of the quality function versus $T_{\text {eff }}$. Good period fits are associated with maxima in the inverse of the quality function.

Unfortunately, there is no clear and unique solution in the range of effective temperatures from spectroscopy; solutions along the cooling tracks for stellar models with masses $M_{\star}=$ $0.584-0.870 M_{\odot}$ all achieve their best fits to the observed periods at these temperatures. However, global best-fit solutions are 
Table 2. Parameters of the model grid used in the fits.

\begin{tabular}{lcc}
\hline \hline Oxygen profile & Helium profile & Other grid parameters \\
\hline$h_{1}=0,1 ; 0.1$ & $M_{\mathrm{env}}=1.5,10 ; 0.5$ & $T_{\mathrm{eff}}=20000-30000 ; 500 \mathrm{~K}$ \\
$h_{2}=0,1 ; 0.1$ & $M_{\mathrm{He}}=M_{\text {env }}, 10 ; 0.5$ & $M_{\star}=0.4,1.0 ; 0.05 M_{\odot}$ \\
$h_{3}=0.8 ;$ fixed & xhe_bar $=0.3 ;$ fixed & ML2 $2 / \alpha=0.96 ;$ fixed \\
$w_{1}=0.02,0.52 ; 0.05$ & $\alpha 1=16 ;$ fixed & No hydrogen $\left(M_{\mathrm{H}}\right.$ fixed to 20) \\
$w_{2}=0.15 ;$ fixed & $\alpha 2=8 ;$ fixed & \\
$w_{3}=0.36 ;$ fixed & & \\
\hline
\end{tabular}

Notes. For a description of each, see Bischoff-Kim \& Montgomery (2018) and Bischoff-Kim (2018). For each parameter, we list the range followed by the step size. $M_{\mathrm{He}}, M_{\mathrm{env}}$, and $M_{\mathrm{H}}$ are defined as unitless, negative log fractions of the star by mass.

Table 3. Best fit parameters, result of simplex search.

\begin{tabular}{|c|c|c|c|c|c|c|c|c|c|}
\hline \multirow{2}{*}{$\begin{array}{l}\text { Fit }(m=0 \text { mode }) \\
1(598 \mathrm{~s})\end{array}$} & \multicolumn{2}{|c|}{$\begin{array}{c}\text { Stellar parameters } \\
T_{\text {eff }}, M_{\star}\end{array}$} & \multicolumn{2}{|c|}{$\begin{array}{c}\text { Envelope parameters } \\
\qquad M_{\mathrm{env}}, M_{\mathrm{He}}\end{array}$} & \multicolumn{3}{|c|}{$\begin{array}{c}\text { Core parameters } \\
h_{1}, h_{2}, w_{1}\end{array}$} & \multirow{2}{*}{$\frac{1 / \chi^{2}\left(1 / s^{2}\right)}{14.2}$} & \multirow{2}{*}{$\begin{array}{r}\sigma_{\text {rms }}(\mathrm{s}) \\
0.283\end{array}$} \\
\hline & $30737 \mathrm{~K}$ & $0.487 M_{\odot}$ & 1.505 & 6.158 & 0.721 & 0.246 & 0.370 & & \\
\hline $2(601 \mathrm{~s})$ & $24546 \mathrm{~K}$ & $0.598 M_{\odot}$ & 1.527 & & 0.459 & 0.405 & $0.5 \mathrm{c}$ & 2.47 & 0.680 \\
\hline $3(605 \mathrm{~s})$ & $29650 \mathrm{~K}$ & $0.499 M_{\odot}$ & 3.595 & 6.411 & 0.595 & 0.123 & 0.375 & 14.7 & 0.279 \\
\hline
\end{tabular}

found at higher temperature for the stellar model with $M_{\star}=$ $0.530 M_{\odot}$, at $T_{\text {eff }}=28844 \mathrm{~K}$ if all the periods are assumed to be $\ell=1$ modes and at $T_{\text {eff }}=28600 \mathrm{~K}$ if the observed periods correspond to a mix of $\ell=1$ and $\ell=2$ modes. A good best-fit solution that is in excellent agreement with the spectroscopic effective temperature derived by Voss et al. (2007) is found for a model with $M_{\star}=0.609 M_{\odot}$ and $T_{\text {eff }}=25595 \mathrm{~K}$. The chemical profiles and Brunt-Väisälä frequency of the model with $M_{\star}=0.530 M_{\odot}$ are plotted in Fig. 11 in the next section, and the best solution for the $M_{\star}=0.609 M_{\odot}$ model that agrees better with the spectroscopic effective temperature is displayed in Fig. 12. If we assume that $f_{3}$ and $f_{5}$ are components of a rotational triplet (thus assuming that they are dipole modes), and consider the average of the periods at $597.6 \mathrm{~s}$ and $604.6 \mathrm{~s}$ in our procedure, then the period fits do not improve substantially.

\subsection{Analysis from the Texas group}

The second asteroseismic fitting analysis that we performed uses models where the chemical profiles are parameterized, along with a few other properties. We used the WDEC (Bischoff-Kim \& Montgomery 2018) with the parameterization of core oxygen profiles described in Bischoff-Kim (2018). In addition to the six core parameters and five parameters describing the helium chemical profile, we can also vary the ML2 mixing length coefficient $\alpha$ (Bohm \& Cassinelli 1971) as well as the mass and effective temperature of the model. A 15 th parameter sets the location of the base of the hydrogen layer, which is not relevant for DBVs.

We had to fix some parameters in order to keep the problem computationally tractable and also constrained. We fixed ML2/ $\alpha$ to 0.96 (see Bischoff-Kim \& Montgomery 2018) and some oxygen and helium profile parameters to values such that we reproduced profiles from Dehner \& Kawaler (1995) and Althaus et al. (2009). Bischoff-Kim (2015) demonstrated that varying the mixing length parameter has a negligible effect on the pulsation periods in the range observed for TIC 257459955. We did allow three of the oxygen profile parameters $\left(h_{1}, h_{2}\right.$, and $\left.w_{1}\right)$ to vary, as well as two of the helium profile parameters (the location of the base of the helium envelope $M_{\mathrm{env}}$ and the pure helium layer mass
Table 4. List of periods for the best fit models.

\begin{tabular}{lccccc}
\hline $\begin{array}{l}\text { Mode } \\
\text { ID }\end{array}$ & k & $\begin{array}{c}\text { Observed period } \\
(\mathrm{s})\end{array}$ & $\begin{array}{c}\text { Fit } 1 \\
(\mathrm{~s})\end{array}$ & $\begin{array}{c}\text { Fit } 2 \\
(\mathrm{~s})\end{array}$ & $\begin{array}{c}\text { Fit 3 } \\
(\mathrm{s})\end{array}$ \\
\hline$f_{9}$ & 4 & 245.4399 & 245.5704 & 245.7442 & 245.4326 \\
$f_{4}$ & 10 & 485.5275 & 485.7931 & 486.3560 & 485.5028 \\
$f_{6}$ & 12 & 557.6493 & 557.3893 & 557.1444 & 558.0835 \\
$f_{3}$ & 13 & 597.5538 & 597.9700 & & \\
Inferred & 13 & 601.1 & & 602.0 & \\
$f_{5}$ & 13 & 604.6423 & & & 604.1388 \\
$f_{1}$ & 14 & 640.5330 & 640.5687 & 641.1684 & 640.7684 \\
$f_{2}$ & 15 & 678.4328 & 678.4701 & 678.0488 & 678.2318 \\
$f_{7}$ & 17 & 749.2676 & 748.9701 & 748.9309 & 749.3228 \\
$f_{8}$ & 20 & 865.9744 & 865.5980 & 865.1238 & 865.9226 \\
\hline$\sigma_{\text {rms }}(\mathrm{s})$ & & \multicolumn{5}{c}{0.283} & 0.680 & 0.279 \\
\hline
\end{tabular}

Notes. All modes are $\ell=1$.

$\left.M_{\mathrm{He}}\right)^{9}$. In addition, we varied the mass and the effective temperature of the models, for a total of seven parameters. These parameters were determined to be the ones that had the greatest effect on the quality of the fits.

We started our model comparison with a grid search to locate minima in the global parameter space. The values of the parameters calculated in our grid are listed in Table 2. In our comparison of the measured periods to the models, we considered three different values for the $m=0$ component in the 598, $605 \mathrm{~s}$ multiplet: we tested each of these as the central component individually, as well as their average, which could be undetected between two observed $m= \pm 1$ modes. We refer to our results from different assumptions of the $m=0$ component of this mode as fits 1-3 in order of increasing period.

We finished with a simplex search (Nelder \& Mead 1965) to refine the minima, calculating WDEC models on the fly as the algorithm sought to minimize $\sigma_{\text {rms. }}$. The simplex method explores the parameter space of the models on its own; it does

9 For shorthand, these helium profile parameters are defined as the negative $\log$ fractions of the star by mass; e.g., $M_{\text {env }}=1.5$ means, in mass units, $M_{\text {env }}=10^{-1.5} \times M_{\star}$. 


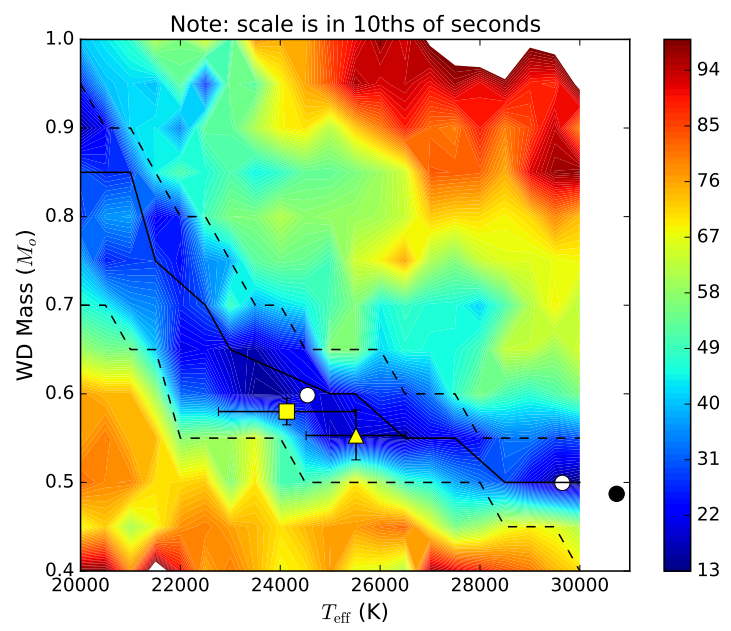

Fig. 9. Goodness-of-fit in the mass- $T_{\text {eff }}$ plane for fit 3 (605 s mode is $m=0$ ) in units of 10th of seconds. The triangle is the location of the spectroscopic values from Voss et al. (2007), and the square is from Rolland et al. (2018), with error bars indicated. The white and black (colored for visibility) filled circles denote the location of the best fits listed in Table 3. The solid line is a line of constant period spacing at $\Delta P=38.1 \mathrm{~s}$ and the dashed lines show the one-second "error bars" around that line.

not reference the models from our initial grid, nor is it bound to the same mass and effective temperature limits. In fact, some of the best-fit models returned are hotter than $30000 \mathrm{~K}$. We list the parameters of the best fit models in Table 3, along with a measure of the quality of fit, computed the same way as in Sect. 4.2 (Eq. (3)) to facilitate comparison with the previous analysis. We also list a different measure of goodness of fit, the standard deviation $\sigma_{\text {rms }}$, because that is the quantity minimized in our grid and simplex searches:

$\sigma_{\mathrm{rms}}=\sqrt{\frac{m}{m-1} * \chi^{2}}$

where again, $m$ is the number of observed periods. We list the periods of the best fit models in Table 4 .

We show the best fit contour map for fit 3 (605 s mode is $m=0)$ in the mass vs. effective temperature parameter plane in Fig. 9, along with the location of the best fits and lines of constant period spacing corresponding to the value derived from the period spectrum of the star. The contour plots for other $m=0$ choices look similar. The period spacing for the models is calculated by fitting a line through the higher $k$ modes $(k=11$ and up) and determining the slope, in the same way we use the linear fit of Fig. 4 to determine one value for the average period spacing present in the pulsation spectrum of the star. The limit of $k=11$ was chosen by visual inspection. Modes of higher radial overtone follow a linear trend closely, and so are reflective of the asymptotic period spacing discussed in Sect. 4.1. The computation of period spacings for the models in the grid are further discussed in Bischoff-Kim et al. (2019). The correlation between the quality of fits and period spacing is striking. This is to be expected for this object with such a tight linear sequence of (assumed) $\ell=1$ modes (Fig. 4).

To determine the location of the spectroscopic points in Fig. 9, we interpolated WDEC models to translate the $\log g$ measurements for the star into mass. We find $M_{\star}=$ $0.553_{-0.027}^{+0.029} M_{\odot}$ for the Voss et al. (2007) measurements and $M_{\star}=0.580_{-0.015}^{+0.014} M_{\odot}$ for the Rolland et al. (2018) values. The slight difference compared to the values inferred by the La Plata
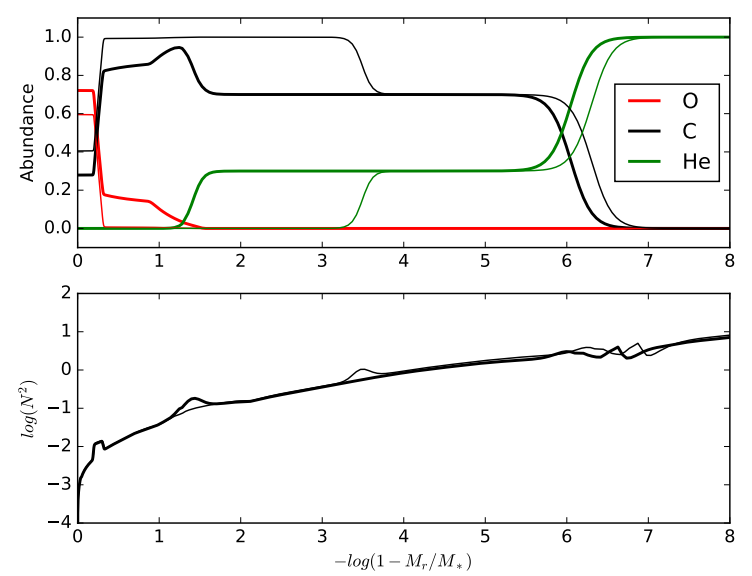

Fig. 10. Top panel: chemical abundance profiles for the two best fit models of Table 3 (fits 1 and 3 with higher effective temperatures). The bold lines correspond to fit 1 , while the thin lines correspond to fit 3 . Bottom panel: corresponding Brunt-Väiäsalä frequency curves.

models comes from the fact that surface gravity depends not only on the mass and effective temperature, but also on the interior structure of the models, and we use different models.

The two best global fits from the simplex search by a significant margin are fit 1 and fit 3. Both are at high effective temperature, inconsistent with the spectroscopic value. They differ mainly by the thickness of the helium envelope, $M_{\text {env }}$. We compare their chemical abundance profiles and Brunt-Väiäsalä frequency curves in Fig. 10. We note a possible manifestation of the core-envelope symmetry here, as has been observed in the asteroseismic fitting of the DBV GD 358 and discussed in Montgomery et al. (2003). The two models differ in the location of bumps in their Brunt-Väiäsalä frequencies corresponding to the transitions from pure carbon to a mix of carbon and helium $\left(\right.$ at $\log \left(1-M_{\mathrm{r}} / M_{\star}\right) \simeq 1.5$ and $\left.\log \left(1-M_{\mathrm{r}} / M_{\star}\right) \simeq 3.5\right)$. The bumps have similar shapes. In the core-envelope symmetry, a feature in the core (or in this case deep in the envelope) can be replaced by a feature further out and produce a similar period spectrum. This will result in two models that fit almost equally well, or in this case a significant change in the location of a Brunt-Väiäsalä feature between best-fit models that use slightly different periods for the $k=13$ mode. The central oxygen abundance and the transition from a mix of helium and carbon to pure helium have a weaker effect on the periods.

Fit 1 agrees closely with the La Plata model that was found to produce the best global period-by-period fit in Sect. 4.2. Considering that we base our fixed parameter values on models from Sect. 4.2, that is expected. We compare the chemical and BruntVäiäsalä profiles of fit 1 with the best-fit La Plata model in Fig. 11. These are similar in the location of the chemical transition zones, which are mainly responsible for setting the period spectrum of a model, so this is not by accident.

In addition, we also have one good fit at lower effective temperature using the average of 598 and $605 \mathrm{~s}$ periods for $m=0$ (fit 2). While this is not nearly as good of a fit to the observed periods, it does agree within uncertainties of both spectroscopic measurements of WD 0158-160's effective temperature. In Fig. 12 we compare the chemical profiles and Brunt-Väiäsalä frequency of this fit to the best-fit solution along the $0.609 M_{\odot}$ evolutionary track from the La Plata models (Sect. 4.2), which also agrees with the spectroscopic $T_{\text {eff }}$. We see again that the features that most affect the period spectrum in the profiles of these cooler secondary solutions appear at roughly the same locations. 

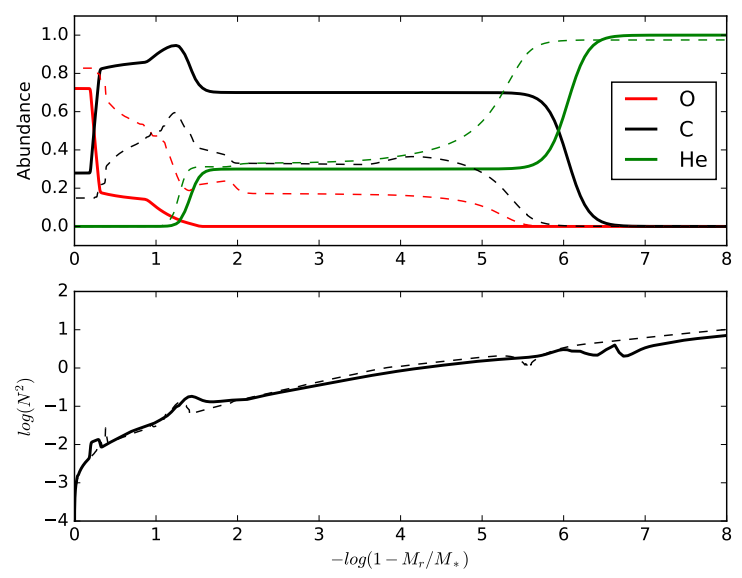

Fig. 11. Top panel: chemical abundance profiles for fit 1 of Table 3 (solid lines), as well as the best fit model of Sect. 4.2 (dashed lines). We chose to contrast these two best fit models because of their similarities. Bottom panel: corresponding Brunt-Väiäsalä frequency curves.

As mentioned in Sect. 4.1, the $598 \mathrm{~s} / 605 \mathrm{~s}$ doublet is consistent with a rotationally split $\ell=1$ mode. The rotation frequency $\Omega$ of the white dwarf is related to the frequency splitting $\Delta \sigma$ by a relation that involves the $m$ identification of the mode and a mode-dependent factor $C_{\mathrm{kl}}$ (Unno et al. 1989):

$\Delta \sigma=m\left(1-C_{\mathrm{kl}}\right) \Omega$.

For our best-fit models we find that the $598 \mathrm{~s}$ mode has $C_{\mathrm{kl}}=$ 0.49 . Using that value and assuming one of the members of the doublet is the $m=0$ mode, we find a rotation period of $7 \mathrm{~h}$. If we have instead observed the $m=1$ and $m=-1$ components of the triplet, then the rotation period is $14 \mathrm{~h}$. Both are consistent with the rotation periods expected empirically for white dwarf stars (Kawaler 2015; Córsico et al. 2019).

\section{Discussion and conclusions}

TESS observed the pulsating helium-atmosphere DBV white dwarf WD $0158-160$ as TIC 257459955 for 20.3 nearly uninterrupted days in Sector 3 at the short two-minute cadence. These data enabled accurate determination of the pulsation frequencies to $\sim 0.01 \mu \mathrm{Hz}$ precision. Our frequency analysis reveals nine significant independent pulsation modes and eleven combination frequencies. The pattern of the observed pulsations is consistent with an incomplete sequence of dipole $\ell=1$ modes with an asymptotic mean period spacing of $38.1 \pm 1.0 \mathrm{~s}$. Two modes separated by $19.6 \mu \mathrm{Hz}$ could belong to a rotationally split $\ell=1$ triplet, implying a stellar rotation period of 7 or 14 hours, depending on which components are being observed.

The shortest-period pulsation at $245 \mathrm{~s}$ was included in our frequency solution based on corroboration with archival photometry from Kilkenny (2016). It appears that a different set of modes were dominant in those ground-based observations that first revealed WD 0158-160 to be a DBV pulsator. Seasonal changes such as these have been observed in other DBVs such as GD 358 (e.g., Bischoff-Kim et al. 2019). The slight residuals in the periodogram of the fully prewhitened time series (Fig. 2) near modes $f_{2}$ and $f_{8}$ likely indicate that these modes were varying in amplitude during the TESS observations.

Enabled by recent improvements in the WDEC (Bischoff-Kim \& Montgomery 2018) and fostered by the collaborative TASC WG8.2, we present for the first time a direct comparison between
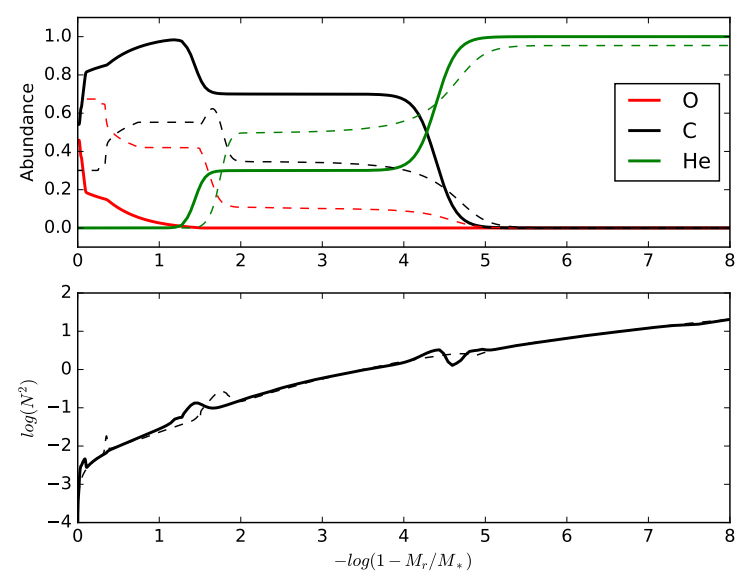

Fig. 12. Top panel: chemical abundance profiles for fit 2 of Table 3 (solid lines), as well as the best fit model for the $0.609 M_{\odot}$ evolutionary track from Sect. 4.2 (dashed lines). These secondary solutions are in better agreement with constraints from spectroscopy. Bottom panel: corresponding Brunt-Väiäsalä frequency curves.

asteroseismic analyses from the La Plata and Texas groups. A primary difference between the two sets of models is that the La Plata group uses fully evolutionary models calculated with LPCODE, while the Texas group computes grids of structural models with parameters sampled on demand using WDEC. Both groups find that the measured mean period spacing of $\ell=1$ modes traces paths of good model agreement of decreasing mass with increasing effective temperature (Figs. 6 and 9) that pass through the average DB white dwarf mass of $\approx 0.62 M_{\odot}$ (e.g., Kepler et al. 2019) at the spectroscopic effective temperature of $25500 \pm 1000 \mathrm{~K}$ from Voss et al. (2007).

When considering individual mode periods, both analyses achieve excellent asteroseismic fits to models with $T_{\text {eff }}$ in excess of $\sim 28500 \mathrm{~K}$ and lower masses $M_{\star} \approx 0.5 M_{\odot}$. These solutions are significantly hotter than the spectroscopic effective temperatures obtained by Voss et al. (2007) and Rolland et al. (2018). External uncertainties in $T_{\text {eff }}$ measured from spectroscopy can be as high as $\approx 1000 \mathrm{~K}$ for DB white dwarfs in the DBV instability strip (e.g., Beauchamp et al. 1999, as assumed in Sect. 4.2), but this is insufficient to bring our optimal seismic fits into agreement with the spectroscopic values. Corrections for spectroscopically determined DB atmospheric parameters based on 3D convection simulations also cannot account for this discrepancy (Cukanovaite et al. 2018). The only DBV reported to have $T_{\text {eff }}>30000 \mathrm{~K}$ is PG $0112+104$, with spectroscopic parameters $T_{\text {eff }}=31300 \pm 500 \mathrm{~K}$ and $M_{\star}=0.52 \pm 0.05 M_{\odot}$ (Dufour et al. 2010) and variability dominated by shorter-period ( 200 s) pulsations (Hermes et al. 2017b).

Both analyses also yield good fits as secondary solutions that are well in line with the spectroscopic measurements. The La Plata evolutionary track for a $0.609 M_{\odot} \mathrm{DB}$ achieves its best fit at $T_{\text {eff }}=25595 \mathrm{~K}$, and WDEC model fit 2 (assuming $601.1 \mathrm{~s}$ for the $k=13$ mode) has $T_{\text {eff }}=24546 \mathrm{~K}$ and a seismic mass of $0.598 M_{\odot}$.

The structural profiles of the global best-fit models from both analyses are compared in Figs. 11 and 12 shows the same for the secondary solutions that agree with spectroscopy. While at first glance, it might appear that there is little agreement between the models from our two analyses, it is important to note that the transition zones (and the corresponding features in the BruntVäiäsalä frequency) do approximately line up. It is well known that pulsation periods are most sensitive to the location of the 
Table 5. Gaia data for the common proper motion stars.

\begin{tabular}{|c|c|c|}
\hline Parameter & TIC 257459955 & G272-B2B \\
\hline RA (deg) & $02^{\mathrm{h}} 00^{\mathrm{m}} 56.8502^{\mathrm{s}}$ & $02^{\mathrm{h}} 00^{\mathrm{m}} 56.9138^{\mathrm{s}}$ \\
\hline Dec (deg) & $-15^{\circ} 46^{\prime} 09.2467^{\prime \prime}$ & $-15^{\circ} 46^{\prime} 16.997^{\prime \prime}$ \\
\hline$G(\mathrm{mag})$ & $14.6789 \pm 0.0018$ & $14.8075 \pm 0.0006$ \\
\hline$G_{\mathrm{BP}}(\mathrm{mag})$ & $14.527 \pm 0.011$ & $16.238 \pm 0.010$ \\
\hline$G_{\mathrm{RP}}(\mathrm{mag})$ & $14.840 \pm 0.008$ & $13.631 \pm 0.002$ \\
\hline Parallax (mas) & $14.64 \pm 0.06$ & $14.55 \pm 0.07$ \\
\hline $\mathrm{RA}$ pm $\left(\mathrm{mas}_{\mathrm{yr}}{ }^{-1}\right)$ & $127.47 \pm 0.10$ & $125.84 \pm 0.12$ \\
\hline $\operatorname{Dec} \mathrm{pm}\left(\mathrm{mas} \mathrm{yr}^{-1}\right)$ & $31.14 \pm 0.08$ & $29.12 \pm 0.10$ \\
\hline Distance $(\mathrm{pc})$ & $68.14 \pm 0.28$ & $68.58 \pm 0.33$ \\
\hline
\end{tabular}

Notes. Distances from Bailer-Jones et al. (2018).

features in the Brunt-Väiäsalä frequency, and less so to their shape (e.g., Montgomery et al. 2003). The consistency of the results of the two seismic analyses is encouraging, as it supports that the results are not dominated by extrinsic errors from the choice of models. Conducting these two analyses in parallel also helps us to select preferred fits for this star.

We can convert the luminosities of our best-fitting models into seismic distances for comparison with the precise astrometric distances available from Gaia DR2 (Gaia Collaboration 2018). Model fit 2 from Table 3 is the WDEC solution with the lowest luminosity $\log L / L_{\odot}=-1.258$ and a bolometric correction (Koster, priv. comm.) of $\mathrm{BC}=-2.55 \mathrm{mag}$. We use the well-known formulas $M_{\mathrm{Bol}}=M_{\mathrm{Bol}, \odot}-2.5 \log \left(L / L_{\odot}\right)$ and $M_{V}=M_{\mathrm{Bol}}-\mathrm{BC}$ to solve for the absolute visual magnitude of $M_{V}=10.44$ for this model, where $M_{\mathrm{Bol}}$ is its absolute bolometric magnitude, and $M_{\mathrm{Bol}, \odot}=4.74$. The apparent visual magnitude of TIC 257459955 from the Fourth US Naval Observatory CCD Astrograph Catalog (Zacharias et al. 2012) is $m_{V}=14.55 \pm 0.08$, which agrees with the Gaia DR2 magnitude of $14.53 \pm 0.01$ in the similar $G_{\mathrm{BP}}$ passband (Gaia Collaboration 2018). Applying the formula $5 \log d=m_{V}+5-M_{V}$, we find that the WDEC model 2 visual magnitude scales to the observed apparent magnitude at a seismic distance of $64.0-68.9 \mathrm{pc}$, which agrees with the Gaia DR2 distance of $68.14 \pm 0.28$ pc from Bailer-Jones et al. (2018) within the error bars. The secondary $0.609 M_{\odot} \mathrm{La}$ Plata solution within the spectroscopic temperature range similarly agrees with the Gaia distance constraint. However, hotter global solutions from both WDEC (models 1 and 3) and LPCODE have temperatures $T_{\text {eff }} \approx 30750-28800 \mathrm{~K}$; the luminosity range is $\log L / L_{\odot}=-0.708$ to -0.867 , which implies a bolometric correction of -3.22 to -3.06 magnitudes. Using the above formulas, these hotter models yield asteroseismic distances 79.8-95.5 pc, which are much further than the distance to TIC 257459955 from Gaia DR2.

Because of their disagreement with the spectroscopic $T_{\text {eff }}$ and the parallax, we regard WDEC models 1 and 3 and the global best-fit LPCODE model as less likely solutions, in spite of their excellent fits to the pulsation periods. We prefer the cooler, secondary solutions from both sets of models that agree with both astrometry and spectroscopy as better representations of TIC 257459955, and we conclude that these external constraints are necessary for selecting the best seismic model given the sensitivity of this particular set of observed modes to the interior stellar structure.

The possibility that the nearby red dwarf G272-B2B is a common proper motion companion to WD $0158-160$, as discussed by Kilkenny (2016), is also supported by the Gaia DR2 astrometric data for these stars, which we summarize in Table 5.
At a distance of $68.58 \pm 0.33$ pc (Bailer-Jones et al. 2018), G272B2B has $M_{V}=9.29$, and using Table 15.7 in Drilling \& Landolt (2000), this absolute magnitude is consistent with a type M1 dwarf, which is a bit more luminous than suggested by Kilkenny (2016). With an on-sky separation of only $7 "$ compared to the TESS plate scale of $21^{\prime \prime}$ pix $^{-1}$, G272-B2B will contribute significant light to the photometric aperture of TIC 257459955. In fact, the header keyword CROWDSAP from the PDC pipeline suggests that only $30 \%$ of the total flux originally measured in the aperture is from the white dwarf target WD 0158-160, which has the effect of decreasing the signal-to-noise of the periodogram by a factor of 1.8 , potentially obscuring lower-amplitude pulsation signals ${ }^{10}$.

We note a striking similarity between the pattern of pulsation modes observed in TIC 257459955 and the prototypical DBV variable GD 358 (Winget et al. 1982). Over three decades of observations have revealed a clear pattern of nearly sequential $\ell=1$ modes in GD 358 with a mean period spacing of $39.9 \mathrm{~s}$ (Bischoff-Kim et al. 2019). This is similar to the period spacing measured from the TESS observations of TIC 257459955, but the periods of the corresponding modes in GD 358 are all longer by $\approx 20$ s. A comparative seismic analysis of these stars could reveal how this relative translation of mode periods results directly from small differentials in their physical stellar parameters.

The pulsation frequencies calculated for stellar models have azimuthal order $m=0$, corresponding to the central components of rotationally split multiplets. Generally, not all components of a multiplet are detected in pulsating white dwarfs, and the observed modes are simply assumed to be $m=0$ in the absence of other information. This can introduce discrete inaccuracies in the fitting of each period of a few seconds, compared to the millisecond precision that these periods are measured to from TESS photometry. The analysis from the Texas group (Sect. 4.3) demonstrated the non-negligible effect of this uncertainty for just a single radial order on the inferred stellar structure, treating each of $f_{3}, f_{5}$, and their average as the $m=0$ mode (Table 3 ). As a manifestation of a core-envelope symmetry, these small changes to a single mode period resulted in significant differences in the best-fit location of the base of the helium envelope in otherwise similar models, as displayed for fits 1 and 3 in Fig. 10. Metcalfe (2003) argued from Monte Carlo tests that fitting models with the assumption of $m=0$ for modes detected from ground-based observations of DBVs yields the same families of solutions and often the same best-fit model (with root-mean-square period differences of $\approx 1 \mathrm{~s}$ ) as when reliable $m$ identifications are available; however, we should be wary of whether these results hold in the era of space photometry, as model fits are now being achieved to the unprecedented precision of our current period measurements (Giammichele et al. 2018).

Our interpretation of the detected signals from the TESS data did not consider their observed amplitudes. We identified the majority of peaks detected in the periodogram as nonlinear combination frequencies that appear at precise differences, sums, and multiples of independent pulsation frequencies (Table 1). These combination frequencies are not comparable to calculations from stellar models and are important to identify and exclude from asteroseismic analyses. The amplitudes detected for the combination signals are expected to be much smaller than the independent pulsation mode amplitudes, and they are typically only detected for combinations of the highest-amplitude modes. We

\footnotetext{
10 The PDC pipeline subtracts off the expected contributions from sources other than the target so that amplitudes measured for detected pulsations should be accurate (Twicken et al. 2010).
} 
find an exception in the presence of a significant peak at the sum of the frequencies of two low-amplitude modes, $f_{4}$ and $f_{5}$, suggesting that $f_{4}+f_{5}$ may actually be an independent pulsation frequency that could improve our asteroseismic constraints. Still, there is less than a $0.5 \%$ chance of an independent mode coinciding this precisely with the sum of any nine other pulsation frequencies. We consider the risk of including a combination frequency in our model comparison much greater that the reward of an ostensibly better but possibly inaccurate fit. The peak at $f_{4}+f_{5}$ would not have been adopted for exceeding our independent significance threshold regardless, and its inclusion as a combination frequency in our solution has a negligible effect on the period measurements for the independent modes. We do not consider the possibility that one of $f_{4}$ or $f_{5}$ may be a difference frequency involving the peak at $f_{4}+f_{5}$, as these individually have higher observed amplitudes and match the pattern of mode frequencies expected from nonlinear pulsation theory.

We note an interesting possibility to test the hypothesis that individual peaks are consistent with combination frequencies based on their relative amplitudes. Wu (2001) provides analytical expressions for the amplitudes of combination frequencies that are based on a physical model for the nonlinear response of the stellar convection zone to the pulsations. This provides a framework for interpreting the amplitudes of combination frequencies in relation to their parent mode amplitudes that could constrain their spherical degrees, $\ell$, and azimuthal orders, $m$ (e.g., Montgomery 2005; Provencal et al. 2012). Besides resolving the common $\ell$ ambiguity when comparing measured to model mode periods, identifying non-axisymmetric modes $(m \neq 0)$ would enable us to apply corrections to our measurements to recover $m=0$ period estimates. This would alleviate the systematic errors from assuming $m=0$ in the model fits, bringing the accuracy of our asteroseimic inferences closer to the level of precision that we currently achieve with the TESS data. Tools to constrain mode identifications in this way for space-based photometry of pulsating white dwarfs are currently in development.

This collaborative first-light analysis from TASC WG8.2 has demonstrated the quality of the TESS observations for measuring pulsations of DBV stars and the current state-of-the-art of their interpretation. TESS is continuing to observe new and known pulsating white dwarfs over nearly the entire sky, providing precise and reliable pulsation measurements for extensive asteroseimic study. Additional first-light papers from TASC WG8.2 on the pulsating PG 1159 star NGC 246 (Sowicka et al.) and an ensemble of DAV stars (Bognár et al.) are in prep.

Acknowledgements. We thank TASC WG8.2 for supporting this project and providing valuable feedback, especially D. Kilkenny and R. Raddi. We thank the anonymous referee whose comments helped to improve this manuscript. KJB is supported by an NSF Astronomy and Astrophysics Postdoctoral Fellowship under award AST-1903828. MHM acknowledges support from NSF grant AST1707419 and the Wootton Center for Astrophysical Plasma Properties under the United States Department of Energy collaborative agreement DE-FOA-0001634. ASB gratefully acknowledges financial support from the Polish National Science Center under project No. UMO-2017/26/E/ST9/00703. ZsB acknowledges the financial support of the K-115709 and PD-123910 grants of the Hungarian National Research, Development and Innovation Office (NKFIH), and the Lendület Program of the Hungarian Academy of Sciences, project No. LP20187/2018. Support for this work was provided by NASA through the TESS Guest Investigator program through grant $80 \mathrm{NSSC} 19 \mathrm{~K} 0378$. This paper includes data collected with the TESS mission, obtained from the MAST data archive at the Space Telescope Science Institute (STScI). Funding for the TESS mission is provided by the NASA Explorer Program. STScI is operated by the Association of Universities for Research in Astronomy, Inc., under NASA contract NAS 5-26555. This work has made use of data from the European Space Agency (ESA) mission Gaia (https://www.cosmos.esa.int/gaia), processed by the Gaia Data Processing and Analysis Consortium (DPAC, https://www. cosmos.esa.int/web/gaia/dpac/consortium). Funding for the DPAC has been provided by national institutions, in particular the institutions participating in the Gaia Multilateral Agreement.

\section{References}

Althaus, L. G., Panei, J. A., Miller Bertolami, M. M., et al. 2009, ApJ, 704, 1605 Althaus, L. G., Córsico, A. H., Isern, J., \& García-Berro, E. 2010, A\&ARv, 18, 471

Astropy Collaboration (Price-Whelan, A. M., et al.) 2018, AJ, 156, 123

Bailer-Jones, C. A. L., Rybizki, J., Fouesneau, M., et al. 2018, AJ, 156, 58 Baran, A. S., Koen, C., \& Pokrzywka, B. 2015, MNRAS, 448, L16

Barentsen, G., Hedges, C., Vinícius, Z., et al. 2019, https://doi.org/10. 5281/zenodo. 2611871

Beauchamp, A., Wesemael, F., Bergeron, P., et al. 1999, ApJ, 516, 887 Bischoff-Kim, A. 2015, 19th European Workshop on White Dwarfs, 175 Bischoff-Kim, A. 2018, https://doi .org/10.5281/zenodo. 1715917 Bischoff-Kim, A., \& Montgomery, M. H. 2018, AJ, 155, 187

Bischoff-Kim, A., Provencal, J. L., Bradley, P. A., et al. 2019, ApJ, 871, 13 Bognár, Z., Paparó, M., Córsico, A. H., Kepler, S. O., \& Győrffy, Á. 2014, A\&A, 570, A116

Bohm, K. H., \& Cassinelli, J. 1971, A\&A, 12, 21

Brickhill, A. J. 1992, MNRAS, 259, 519

Charpinet, S., Brassard, P., Fontaine, G., et al. 2019, A\&A, in press, https: //doi.org/10.1051/0004-6361/201935395

Córsico, A. H., \& Althaus, L. G. 2006, A\&A, 454, 863

Córsico, A. H., Miller Bertolami, M. M., Althaus, L. G., Vauclair, G., \& Werner, K. 2007, A\&A, 475, 619

Córsico, A. H., Althaus, L. G., Miller Bertolami, M. M., \& Bischoff-Kim, A. 2012, A\&A, 541, A42

Córsico, A. H., Althaus, L. G., Miller Bertolami, M. M., Kepler, S. O., \& GarcíaBerro, E. 2014, JCAP, 8, 054

Córsico, A. H., Althaus, L. G., Miller Bertolami, M. M., et al. 2019, A\&ARv, 27,7

Cox, J. P. 1984, PASP, 96, 577

Cukanovaite, E., Tremblay, P.-E., Freytag, B., et al. 2018, MNRAS, 481, 1522

Dehner, B. T., \& Kawaler, S. D. 1995, ApJ, 581, L33

Drilling, J. S., \& Landolt, A. U. 2000, in Allen's Astrophysical Quantities, 4th edn., ed. A. N. Cox (New York, NY: Springer-Verlag), 381

Dufour, P., Desharnais, S., Wesemael, F., et al. 2010, ApJ, 718, 647

Dziembowski, W. 1977, Acta Astron., 27, 203

Fontaine, G., \& Brassard, P. 2008, PASP, 120, 1043

Gaia Collaboration (Prusti, T., et al.) 2016, A\&A, 595, A1

Gaia Collaboration (Brown, A. G. A., et al.) 2018, A\&A, 616, A1

Giammichele, N., Charpinet, S., Fontaine, G., et al. 2018, Nature, 554, 73

Handler, G., Pikall, H., O’Donoghue, D., et al. 1997, MNRAS, 286, 303

Hermes, J. J., Gänsicke, B. T., Kawaler, S. D., et al. 2017a, ApJS, 232, 23

Hermes, J. J., Kawaler, S. D., Bischoff-Kim, A., et al. 2017b, ApJ, 835, 277

Kawaler, S. D. 1988, Advances in Helio- and Asteroseismology, 123, 329

Kawaler, S. D. 2015, 19th European Workshop on White Dwarfs, 65

Kepler, S. O., Pelisoli, I., Koester, D., et al. 2019, MNRAS, 486, 2169

Kilkenny, D. 2016, MNRAS, 457, 575

Metcalfe, T. S. 2003, Balt. Astron., 12, 247

Montgomery, M. H. 2005, ApJ, 633, 1142

Montgomery, M. H., \& O’Donoghue, D. 1999, Delta Scuti Star Newsletter, 13, 28

Montgomery, M. H., Metcalfe, T. S., \& Winget, D. E. 2003, MNRAS, 344, 657

Nelder, J. A., \& Mead, R. 1965, Comput. J., 7, 308

Newville, M., Otten, R., Nelson, A., et al. 2018, https://doi .org/10.5281/ zenodo. 1699739

O’Donoghue, D. 1994, MNRAS, 270, 222

Provencal, J. L., Montgomery, M. H., Kanaan, A., et al. 2012, ApJ, 751, 91

Ricker, G. R., Winn, J. N., Vanderspek, R., et al. 2014, Proc. SPIE, 9143, 914320

Rolland, B., Bergeron, P., \& Fontaine, G. 2018, ApJ, 857, 56

Stumpe, M. C., Smith, J. C., Van Cleve, J. E., et al. 2012, PASP, 124, 985

Tassoul, M., Fontaine, G., \& Winget, D. E. 1990, ApJS, 72, 335

Twicken, J. D., Chandrasekaran, H., Jenkins, J. M., et al. 2010, Proc. SPIE, 7740, $77401 \mathrm{U}$

Unno, W., Osaki, Y., Ando, H., et al. 1989, Nonradial Oscillations of Stars (Tokyo: University of Tokyo Press)

Voss, B., Koester, D., Napiwotzki, R., Christlieb, N., \& Reimers, D. 2007, A\&A, 470, 1079

Winget, D. E., \& Kepler, S. O. 2008, ARA\&A, 46, 157

Winget, D. E., Robinson, E. L., Nather, R. D., et al. 1982, ApJ, 262, L11

Winget, D. E., Nather, R. E., Clemens, J. C., et al. 1994, ApJ, 430, 839

Wu, Y. 2001, MNRAS, 323, 248

Zacharias, N., Finch, C. T., Girard, T. M., et al. 2012, VizieR Online Data Catalog: I/322 